\title{
Treatment of Surgical Site Infection in Posterior Lumbar Interbody Fusion
}

\author{
Jung Su Lee, Dong Ki Ahn, Byung Kwon Chang, Jae Il Lee \\ Department of Orthopedic Surgery, Seoul Sacred Heart General Hospital, Seoul, Korea
}

Study Design: A retrospective observational and case control study.

Purpose: To identify appropriate treatment options according to the types of surgical site infections (SSI) in instrumented posterior lumbar interbody fusion (PLIF).

Overview of Literature: There has been no agreement or consensus with regard to this matter.

Methods: Thirty-two consecutive SSIs were included and followed for more than one year. The elapsed time to diagnosis (ETD) according to the type of SSI was analyzed. The treatment options for each type and consequent clinical results were reviewed. The risk factors of removing the implants were analyzed.

Results: There were 6/32 (19\%) superficial incisional, 6/32 (19\%) deep incisional, and 20/32 (62\%) organ/space infection cases (SII, DII, and 0/SI, respectively) ( $p=0.002$ ). ETD was $8.5 \pm 2.3$ days in SII, $8.7 \pm 2.3$ days in DII, and $164.5 \pm 131.1$ days in $0 / S I$ ( $p=0.013$ ). All cases of SII and DII retained implants and were treated by repeated irrigation and secondary closure. Among 0/Sls, 10/20 were treated conservatively. Nine out of ten underwent posterior one stage simultaneous revision (POSSR) and in one case, the cage was removed anteriorly. Those who had ETDs longer than 3 months showed a significant risk of implant removal $(p=0.008$, odds ratio $[\mathrm{OR}]=40.3$ ). The Oswestry disability index (ODI) improved from $47.3 \%$ to $33.8 \%$ in SII, from $55.0 \%$ to $32.3 \%$ in DII, and from $53.4 \%$ to $42.1 \%$ in $0 / S I(p=0.002)$. There was no difference among the three groups $(p=0.106)$; however, there was a partial correlation between ETD and final ODI $(r=0.382, p=0.034)$.

Conclusions: Latent O/SI was the most common type of SSI in PLIF. In cases of SII and DII, early aggressive wound management and secondary closure was effective and implant removal was not necessary. In some cases of $0 / \mathrm{SI}$, implant removal was unavoidable. However, implant removal could be averted by an earlier diagnosis. POSSR was feasible and safe. Functional outcomes were improved; however, disability increased as ETD increased.

Keywords: Surgical site infection; Posterior lumbar interbody fusion; Treatment; Implant removal; Posterior one stage simultaneous revision

\section{Introduction}

In terms of simultaneous anterior reconstruction and high union rate, posterior lumbar interbody fusion (PLIF) has many advantages over posterolateral fusion (PLF)
[1-4]. However, at the same time, it has more complications. Surgical site infections (SSIs) develop more often in PLIF, with varying types. Spondylitis around interbody cages and grafted bone were frequent in our cases. It is more difficult to decide whether to preserve or remove

\footnotetext{
Received Apr 21, 2015; Revised May 15, 2015; Accepted May 16, 2015

Corresponding author: Dong Ki Ahn

Department of Orthopedic Surgery, Seoul Sacred Heart General Hospital,

Wangsanrho 259, Dongdaemun-gu, Seoul 02488, Korea

TEL: +82-2-966-1616, FAX: +82-2-968-2394, E-mail: adkajs@hanmail.net
} 
the implant in those situations because cage removal is technically demanding and results in total segmental instability and neurological compromise. As far as we know, there has been no study about specific treatments for SSIs in PLIF. We have classified different SSIs in PLIF and analyzed results according to the respective types and treatment methods. Furthermore, risk factors of implant removal were studied.

\section{Materials and Methods}

We performed a retrospective observational and case control study in which those who were treated for SSI after PLIF in our institute between 2005 and 2012 and follow for at least 12 months were included. Diagnosis and classification followed the criteria of Center for Disease Control and Prevention (CDC) of the United States. In multi-segment surgeries, PLIF segments that were the same or more than half were regarded as PLIF. Demography, elapsed time to diagnosis (ETD), causative microorganisms, methods of treatment, and their results were investigated and compared according to the classification. The time of diagnosis was defined as when exudate from a surgical incision was found in superficial and deep incisional infections (SII and DII, respectively) and when antibiotics were administered because of laboratory and image findings that suggested organ/space infection $(\mathrm{O} / \mathrm{SI})$. In the treatment, the duration of intravenous antibiotics administration and elapsed time to a secondary closure were investigated. Intravenous antibiotics were continued to the time of two consecutive normal range measurements of C-reactive protein (CRP). A secondary closure was implemented when the wound was clear on a visual examination and microorganisms were not identified on a microbiological exam. Risk factors of implant removal were investigated, including age, sex, diabetes mellitus, ETD, and radiological implant loosening. Successful treatment was defined as no more clinical, laboratory, and image finding of infection. Bone union state and functional state using the Oswestry disability index (ODI) were investigated and the differences according to the types and influencing factors were analyzed. For the statistical analysis, T tests, Fisher's exact tests and analysis of variance were used to prove the difference among the types and a logistic regression test was used for the risk factors of implant removal. Significance was defined as $p \leq 0.05$ and SPSS ver. 16.0 (SPSS Inc., Chicago, IL, USA) package was used for all analyses.

\section{Results}

There were 32 cases of SSI. Thirty out of 1,381 cases (1.6\%) developed at author's hospital and two cases were transferred in from other hospitals. The average age of patients was 64 years (range, 41-91 years) and there were 16 males and 16 females. The number of fusion segments was 1 in 21 cases (65.6\%), 2 in 9 cases (28.1\%) and 3 in 2 cases (6.3\%). Diabetes mellitus was present in 8 cases (25\%). According to the CDC classification, there were 6 cases (18.8\%) of SII, 6 cases (18.8\%) of DII, and 20 cases $(62.5 \%)$ of O/SI. All O/SIs were spondylitis. The incidence of each type was significantly different $(p=0.002)$. Ten cases (31\%) had implant loosening with both pedicle screws and cages at the time of diagnosis. Ten cases did not have microbiological exams. Among the 22 cases that did undergo microbiological exams, 6 cases showed no growth. There were 6 cases of methicillin-resistant Staphylococcus epidermidis (MRSE), 5 cases of methicillin-sensitive Staphylococcus epidermidis (MSSE), 2 cases of methicillinresistant Staphylococcus aureus (MRSA), and 1 case each of Escherichia coli, methicillin-sensitive Staphylococcus aureus (MSSA), and Staphylococcus pyogenes. All SSIs $(6 / 6)$ and 5/6 DIIs were treated by repeated irrigation and a secondary closure. The remaining DII underwent vacuum dressing and a secondary closure. Ten out of 20 (50\%) cases of O/SI were treated conservatively (Fig. 1)

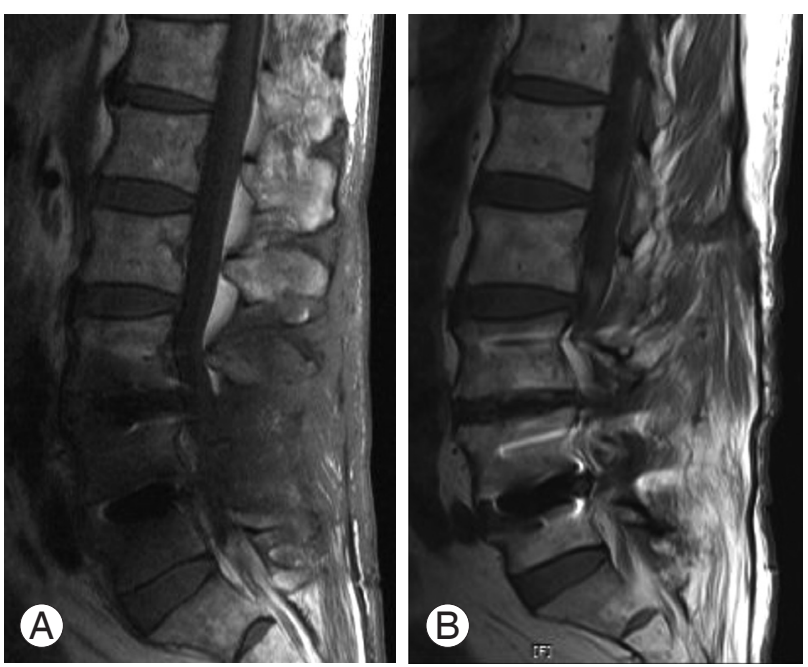

Fig. 1. (A) T1-weighted magnetic resonance (MR) image showing a low signal change around L45 interbody cages that suggests spondylitis. However, there is no draining sinus or pus collection in the posterior incisional route. (B) T1-weighted MR image of the same patient after 3 months of conservative treatment. The low signal change was completely recovered. 
Table 1. Demography and results

\begin{tabular}{|c|c|c|c|c|c|c|c|c|c|c|}
\hline Case & Sex & Age (yr) & Class & ETD & Microbiology & $\mathrm{Lx}$ & Treatment & IVA & ODI & Union \\
\hline 1 & Male & 51 & SII & 7 & MRSE & - & Irr \& 2nd C & 23 & 35 & + \\
\hline 2 & Female & 54 & SII & 6 & S. epidermidis & - & Irr \& 2nd C & 22 & 29 & + \\
\hline 3 & Male & 58 & SII & 7 & MRSE & - & Irr \& 2nd C & 20 & 36 & + \\
\hline 4 & Female & 70 & SII & 10 & MRSE & - & Irr \& 2nd C & 10 & 40 & + \\
\hline 5 & Male & 72 & SII & 12 & MRSA & - & Irr \& 2nd C & 20 & 34 & + \\
\hline 6 & Female & 74 & SII & 9 & MRSA & - & Irr \& 2nd C & 27 & 29 & + \\
\hline 7 & Male & 57 & DII & 7 & S. epidermidis & - & Irr \& 2nd C & 57 & 35 & + \\
\hline 8 & Male & 62 & DII & 6 & MRSE & - & Irr \& 2nd C & 37 & 27 & + \\
\hline 9 & Female & 65 & DII & 10 & Not identified & - & Irr \& 2nd C & 30 & 29 & + \\
\hline 10 & Female & 71 & DII & 10 & S. epidermidis & - & Irr \& 2nd C & 63 & 31 & + \\
\hline 11 & Male & 83 & DII & 7 & MRSE & - & Irr \& 2nd C & 71 & 30 & + \\
\hline 12 & Female & 41 & DII & 12 & Not identified & - & Revision & 22 & 42 & + \\
\hline 13 & Female & 54 & O/SI & 270 & Not identified & + & Revision & 28 & 38 & + \\
\hline 14 & Female & 56 & 0/SI & 86 & No culture & - & Conservative & 50 & 41 & + \\
\hline 15 & Male & 58 & $0 / \mathrm{SI}$ & 38 & No culture & - & Conservative & 29 & 25 & + \\
\hline 16 & Female & 60 & O/SI & 90 & No culture & - & Conservative & 81 & 42 & + \\
\hline 17 & Male & 60 & 0/SI & 370 & MRSE & - & Revision & 77 & 51 & + \\
\hline 18 & Female & 61 & 0/SI & 410 & S. aureus & + & Revision & 28 & 48 & + \\
\hline 19 & Male & 64 & 0/SI & 60 & No culture & + & Conservative & 29 & 65 & - \\
\hline 20 & Female & 68 & O/SI & 30 & No culture & - & Conservative & 55 & 22 & + \\
\hline 21 & Male & 68 & O/SI & 10 & No culture & - & Conservative & 56 & 28 & + \\
\hline 22 & Male & 70 & 0/SI & 180 & Not identified & + & Revision & 31 & 38 & + \\
\hline 23 & Male & 71 & 0/SI & 95 & Not identified & + & Revision & 45 & 45 & + \\
\hline 24 & Male & 72 & 0/SI & 50 & No culture & - & Conservative & 30 & 28 & + \\
\hline 25 & Female & 73 & $0 / \mathrm{SI}$ & 25 & S. pyogenes & - & Revision & 80 & 45 & + \\
\hline 26 & Female & 74 & O/SI & 240 & Escherichia coli & + & Revision & 28 & 67 & + \\
\hline 27 & Male & 74 & $0 / S I$ & 300 & Not identified & + & Revision & 46 & 33 & + \\
\hline 28 & Female & 53 & 0/SI & 270 & No culture & + & Conservative & 56 & 67 & + \\
\hline 29 & Female & 63 & $0 / S I$ & 240 & No culture & - & Conservative & 42 & 30 & + \\
\hline 30 & Male & 65 & O/SI & 10 & No culture & - & Conservative & 49 & 53 & - \\
\hline 31 & Female & 66 & $0 / \mathrm{SI}$ & 180 & S. epidermidis & + & Revision & 25 & 36 & + \\
\hline 32 & Male & 67 & $0 / \mathrm{SI}$ & 335 & S. epidermidis & + & Revision & 42 & 34 & + \\
\hline
\end{tabular}

ETD, elapsed time to a diagnosis; Lx, radiological loosening; IVA, intravenous antibiotics; ODI, Oswestry disability index; SII, superficial incisional infection; MRSE, methicillin resistant Staphylococcus epidermidis; Irr \& 2nd C, irrigation and secondary closure; S. epidermidis, Staphylococcus epidermidis; MRSA, methicillin resistant Staphylococcus aureus; DII, deep incisional infection; 0/SI, organ/space infection; S. aureus, Staphylococcus aureus; S. pyogenes, Staphylococcus pyogenes.

and 10/20 (50\%) cases received revision surgeries. When four weeks of intravenous antibiotics did not show any response, or when neurological symptoms developed due to instability or cage retropulsion or epidural abscess, surgery was performed. The elapsed time to secondary closure was 9 days (range, 8-13 days) in SII and 26 days (range, 10-46 days) in DII. The difference was significant $(p=0.033)$. The duration of intravenous antibiotics was 20 days (range, 10-27 days) days in SII, 45 days (range, 11-71 days) in DII, and 45 days (range, 23-81 days) in 
$\mathrm{O} / \mathrm{SI}$. The difference between SII and DII $(p=0.030)$ and between SII and O/SI $(p=0.009)$ was significant, but the difference between DII and O/SI was not significant $(p=0.985)$. In the subgroup analysis of $\mathrm{O} / \mathrm{SI}$, the difference was not significant $(p=0.987)$ between the conservative treatment group (48 days; range, 29-81 days) and the revision group (43 days; range, $25-80$ days) (Table 1 ). In the revision group, $9 / 10$ cases underwent posterior one stage simultaneous revision (POSSR). All implants including cages were removed. All infected tissues were excised and the interbody space was irrigated copiously. Bi-cortical auto-iliac bone blocks were grafted in the interbody space. Re-implantation was performed. Widened pedicle holes were charged with fresh frozen allograft bone chips and fusion segments were extended if needed. However, there was one case of a posterior cage removal failure. In that case, auto-iliac bone chips were grafted while leaving the cage (Fig. 2). One case underwent anterior cage removal and tricortical auto-iliac bone was grafted while preserving the posterior instrumentation and a hip spica cast was applied for 6 weeks. In the final analysis, all cases were cured of their respective infections. SII and DII were cured without implant removal. Two out of $10 \mathrm{O} / \mathrm{SI}$ that were treated conservatively ended up with nonunion. One of them showed retropulsion of the cage; however, the patient did not develop neurological symptoms and showed normalized laboratory findings. All revision cases $(10 / 10)$ had bone union. Ultimately, 22 cases preserved their implants while 10 cases failed to do so. Risk factors of implant removal included the implant loosening and an ETD longer than 3 months in univariable analysis. However, in a multivariable logistic regression test, only ETD longer than 3 months was recognized as a risk factor $(p=0.023, \mathrm{OR}=32.592)$ (Table 2$)$. Three months was determined by an ROC curve as the time that resulted in the widest area under the curve. Functional results as measured by ODI were improved from pre-treatment levels of $53.1 \%$ to $38.5 \%$ as a whole $(p<0.001)$. SII improved from $47.3 \%$ to $33.8 \%$ ( $p=0.028$ ), DII improved from $55.0 \%$ to $32.3 \%$ ( $p=0.043$ ), and $\mathrm{O} / \mathrm{SI}$ improved from $53.4 \%$ to $43.5 \%$ ( $p=0.003$ ). The ODI of O/SI was lower, but it was not statistically significant $(p=0.175)$. In the subgroup analysis of O/SI, there was no difference of ODI between the conservative and revision groups, which were $40.1 \%$ and $43.5 \%$, respectively $(p=0.344)$. There was a correlation between the ETD and final ODI while considering the confounding effect of preoperative ODI $(r=0.374, p=0.038)$.

\section{Discussion}

The most prominent feature of SSI in PLIF was high incidence of O/SI. O/SI is known to have a long ETD. According to the definition of the $\mathrm{CDC}, \mathrm{O} / \mathrm{SI}$ is defined as an SSI which involves any part of anatomy which was opened and manipulated during an operation other than the incision itself [5]. The common features of SII and DII were not so different from other types of posterior instrumented surgery. However O/SI, i.e., spondylitis without
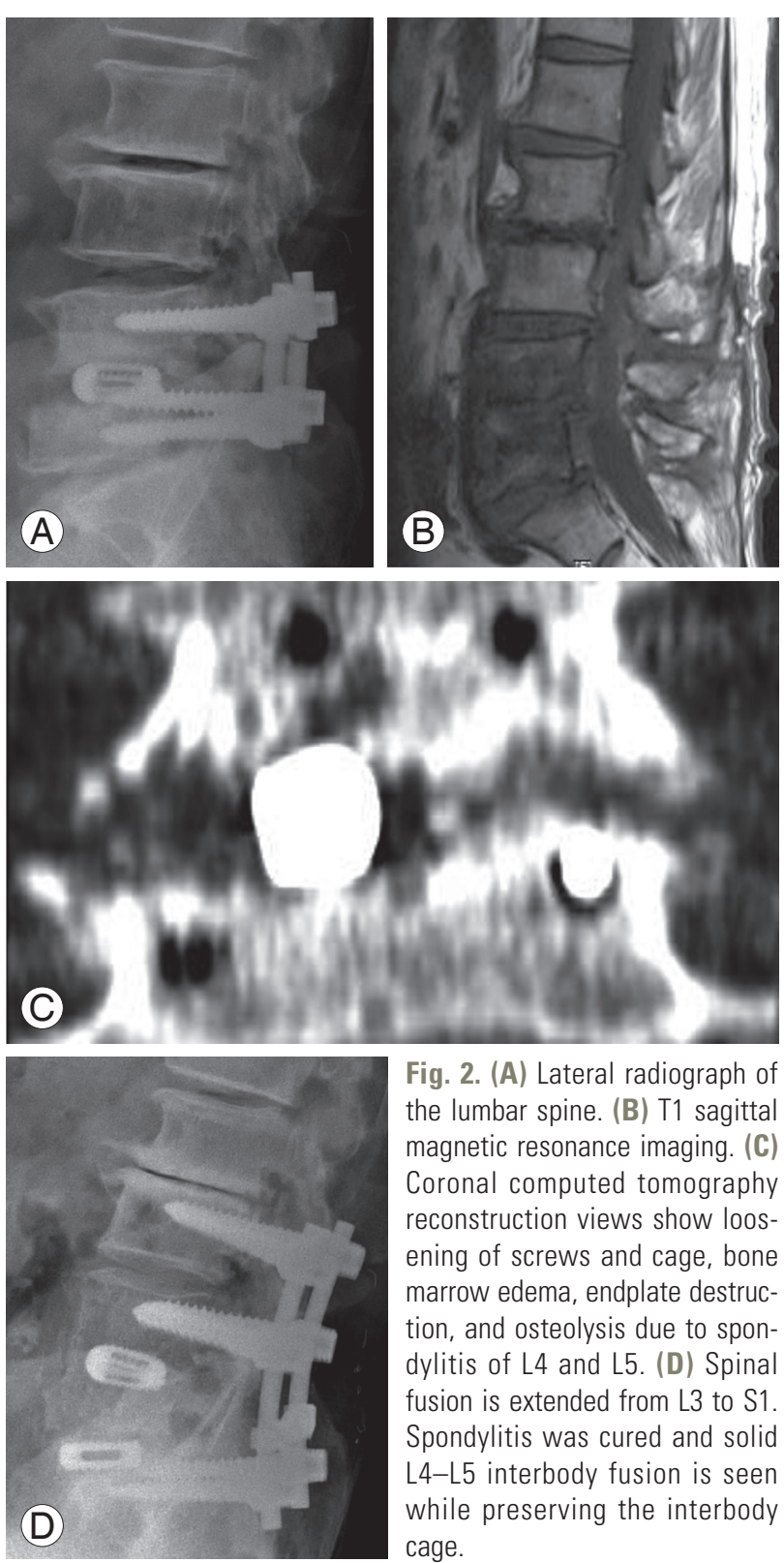

Fig. 2. (A) Lateral radiograph of the lumbar spine. (B) T1 sagittal magnetic resonance imaging. (C) Coronal computed tomography reconstruction views show loosening of screws and cage, bone marrow edema, endplate destruction, and osteolysis due to spondylitis of L4 and L5. (D) Spinal fusion is extended from L3 to S1. Spondylitis was cured and solid L4-L5 interbody fusion is seen while preserving the interbody cage. 
Table 2. Risk factors for implant removal

\begin{tabular}{|c|c|c|c|c|}
\hline \multirow[b]{2}{*}{ Factor } & \multirow[b]{2}{*}{ Implant retaining } & \multirow[b]{2}{*}{ Implant removal } & \multicolumn{2}{|c|}{$p$-value } \\
\hline & & & Univariate & $\begin{array}{l}\text { Multivariate } \\
\text { (odds ratio) }\end{array}$ \\
\hline Age (yr) & $63.0 \pm 9.3$ & $67.0 \pm 6.8$ & 0.226 & - \\
\hline Sex (male/female) & $11 / 11$ & $5 / 5$ & 1.000 & - \\
\hline Diabetes mellitus ( \pm ) & $5 / 17$ & $3 / 7$ & 0.661 & - \\
\hline Elapsed time to a diagnosis ( $>3 \mathrm{mo} /<3 \mathrm{mo}$ ) & $20 / 2$ & $1 / 9$ & 0.000 & $0.023(32.592)$ \\
\hline Ra-loosening $( \pm)^{\text {a) }}$ & $2 / 20$ & $8 / 2$ & 0.001 & 0.297 \\
\hline Total & 22 & 10 & - & - \\
\hline
\end{tabular}

The bold letters mean statistical significance.

a) Radiological loosening.

incisional infection, has a range of different features from diagnosis to treatment. In the case of incisional infections, implant removal was not necessary. They were all diagnosed early and, consequently, local management began as soon as possible. Wound exploration and repeated irrigation was considered effective to prevent the infection's extension to deeper structures. Fortunately, there was no bone extension from incisional infections. Many authors have recommended the retention of implants in acute infections [6-10]. However, in other studies, implant removal was frequently requested in DII [11-14]. In particular, late onset infections and Propionibacterium acne (PA) infections were found to be risk factors [13]. There was no late onset infection in the incisional infections in our cases. This is a possible reason why we were able to preserve all implants in the incisional infections. O/SI was not easily diagnosed because of its vague symptoms and an absence of local findings. Therefore, the ETD was further delayed after the true onset of infection in those cases. The O/SI almost always began from the interbody space as a spondylitis around the cages and grafted bone. It was difficult to presume that bacteria contaminated the interbody space only. Rather, it would be more plausible to presume that contaminated bacteria located in the interbody space were not easily removed by irrigation and adhered to disc remnants or foreign materials, and they gradually developed a late infection. If the contaminated bacteria were highly virulent, it is presumed that a more aggressive and acute onset infection would develop. In our cases, half of them did not undergo microbiological exams because they were managed conservatively. Intravenous antibiotics were started when laboratory and clini- cal manifestations strongly suggested infection. Cefolactam (Samjin Pharm, Seoul, Korea) that was comprised of cefoperazone and sulbactam was used as the intravenous antibiotic. Antibiotics were switched once the causative microorganism was determined from the sensitivity test. Among the 10 cases which underwent revision surgeries, there were no highly virulent microorganisms. Four cases showed no growth. There have been many studies that noted disc space infections after discectomy by anaerobic bacteria, especially PA [15-19], and the late infection in instrumented spinal surgeries by PA $[13,20,21]$. Disc space contamination by PA has been proven through other studies $[22,23]$. We did not perform anaerobic culturing at first and did perform it later on. PA was not identified in any case; however, some of the cases might have been PA infections. The remaining cases were also not highly virulent infections. At first, we could not appropriately diagnose O/SI. After we became aware of its clinical manifestations, the ETD was shortened. Furthermore, we realized that conservative treatment was possible if an early diagnosis was made. Maruo and Berven [13] also noted that a late infection, in other words, late diagnosis, was a risk factor for treatment failure. The preservation of implants seriously matters in O/SI because cage removal is technically difficult [12]. Furthermore the index segment would lose stability entirely and neurological symptoms could develop after implant removal. If staged operations were performed, patients had to stay on bed rest during the interval. That is why we could not prevent performing POSSR. As far as we have researched, there is no agreement for the treatment for infections such as O/SI. Carmouche and Molinari [11] tried to preserve the cages 
but ultimately ended up removing the cages and achieved spinal fusion by posterolatral bone grafts. Ha and Kim [12] concluded that all implants should be removed to counter any spondylitis around the cages and that the anterior approach was feasible to remove them. Sierra-Hoffman et al. [14] and Hedequist et el. [24] determined that, in cases caught early, the implants could be preserved, but that late cases necessitated implant removal. There are many presumptive risk factors of implant removal. However, an ETD longer than 3 months was the only independent risk factor in our study. All revision cases had implant loosening. In an image test; however, two of them appeared not having loosening. For that reason, radiological implant loosening was not counted as an independent risk factor. Therefore, a keen suspicion is the first and most important step for the successful diagnosis of an SSI, which is directly related to successful treatment. In revision surgeries, all screws and cages were loosened and grafted bone chips were already sequestrated. Cage removal is a demanding procedure, but it is not impossible. Before cage removal, all screws and rods were removed and widened holes were charged with fresh frozen allograft bone chips and bigger screws were inserted. The interbody space was distracted to make a space for the cage removal. However, we failed to remove the cage in one case. In that case, we displaced it to the opposite side and grafted an auto-iliac bone. At the final assessment, the patient achieved solid interbody fusion and the cage was embedded in the bone mass. There have been many reports stating that titanium cages can be used in the surgical treatment of spondylitis [25-28]. We are unsure if titanium cages are safe to use in spondylitis or not. Furthermore, the titanium cage would be different from the initially applied cage because it was presumed to be covered by a biofilm of microorganisms. Though our case cannot be generalized, the meticulous debridement and copious irrigation with a pulsatile irrigator of the interbody space and auto-iliac bone grafts were considered more important than the titanium cage removal itself. While all revision cases achieved interbody fusion, two cases of spondylitis that underwent conservative treatment resulted in a nonunion state. One patient was asymptomatic, while the other had discomfort and back pain aggravated by motion but refused to receive a revision. If the causative bacteria were highly virulent, the treatment options would have been different. Tokuhashi et al. [29] reported the successful treatment of spondylitis around cages without surgical intervention, though there was already an epidural abscess and explained it was possible because the causative bacteria were lowly virulent. POSSR might have been dangerous if the SSI was caused by highly virulent bacteria. POSSR has several drawbacks. Posterior interbody debridement might be incomplete and bicortical auto-iliac bone from the posterior iliac crest is not as strong as tricortical bone from the anterior iliac crest. It has, however, many advantages. Through a posterior approach, all implants and infected tissues in the epidural and interbody spaces could be removed. Furthermore, stability could be attained under the same anesthesia and without positional changes, minimizing neurological injury and reducing the patient's inconvenience which would result from a longer duration bed rest. At first, we thought the widened pedicle holes should be charged with auto-iliac bone. But the amount of auto-iliac bone was not enough to cover both the interbody space and the pedicle holes. Furthermore, it did not have sufficient structural hardness, due to osteoporosis. So, we cautiously used fresh frozen allogeneic bone from the bone bank of author's institute. Fortunately, there have been no adverse effects so far. Functional results were improved after treatment of the SSIs in general. Though functional improvements were not as strong in $\mathrm{O} / \mathrm{SI}$, it did not reach statistical significance. O/SI was influenced by ETD. We could say SII and DII had better functional results than $\mathrm{O} / \mathrm{SI}$; however, the early diagnosed O/SI was not inferior to SII or DII. Therefore, an early diagnosis was the key to averting implant removal and attaining a better functional result. There were several limitations in our study. As a general limitation of observational studies, the results of our case series could not represent every specific situation. In particular, our cases were mainly caused by low virulent microorganisms. A more precautious strategy would be necessary for the highly virulent aggressive SSI.

\section{Conclusions}

The incidence of SSI in PLIF was 1.6\%. O/SI was the most common type. In SII and DII, implant removal was not necessary and early wound exploration and repeated irrigation followed by secondary closure was an effective treatment regimen. In $\mathrm{O} / \mathrm{SI}$, however, $50 \%$ of patients needed implant removal, though the early diagnosed cases could be treated conservatively. ETDs longer than 3 months conspicuously increased the risk of implant re- 
moval. POSSR, from implant removal to re-implantation, was effective for the spondylitis around interbody cages. It minimized patients' discomfort by avoiding a staged operation or dual approaches. Functional results were improved as compared to the pre-treatment state, but were not distinguishable from type to type. However, a long ETD made it worse. Therefore, a keen suspicion to detect vague clues and an early diagnosis followed by prompt and appropriate treatments were the most important in treating SSI in PLIF.

\section{Conflict of Interest}

No potential conflict of interest relevant to this article was reported.

\section{References}

1. Liu X, Wang Y, Qiu G, Weng X, Yu B. A systematic review with meta-analysis of posterior interbody fusion versus posterolateral fusion in lumbar spondylolisthesis. Eur Spine J 2014;23:43-56.

2. Fritzell P, Hagg O, Wessberg P, Nordwall A. Chronic low back pain and fusion: a comparison of three surgical techniques: a prospective multicenter randomized study from the Swedish lumbar spine study group. Spine (Phila Pa 1976) 2002;27:1131-41.

3. Christensen FB, Hansen ES, Eiskjaer SP, et al. Circumferential lumbar spinal fusion with Brantigan cage versus posterolateral fusion with titanium Cotrel-Dubousset instrumentation: a prospective, randomized clinical study of 146 patients. Spine (Phila Pa 1976) 2002;27:2674-83.

4. Schofferman J, Slosar P, Reynolds J, Goldthwaite N, Koestler M. A prospective randomized comparison of 270 degrees fusions to 360 degrees fusions (circumferential fusions). Spine (Phila Pa 1976) 2001;26: E207-12.

5. Horan TC, Gaynes RP, Martone WJ, Jarvis WR, Emori TG. CDC definitions of nosocomial surgical site infections, 1992: a modification of CDC definitions of surgical wound infections. Am J Infect Control 1992;20:271-4.

6. Ahmed R, Greenlee JD, Traynelis VC. Preservation of spinal instrumentation after development of postoperative bacterial infections in patients undergoing spinal arthrodesis. J Spinal Disord Tech 2012;25:299-
302.

7. Gerometta A, Rodriguez Olaverri JC, Bitan F. Infections in spinal instrumentation. Int Orthop 2012;36: 457-64.

8. Mirovsky Y, Floman Y, Smorgick Y, et al. Management of deep wound infection after posterior lumbar interbody fusion with cages. J Spinal Disord Tech 2007;20:127-31.

9. Pappou IP, Papadopoulos EC, Sama AA, Girardi FP, Cammisa FP. Postoperative infections in interbody fusion for degenerative spinal disease. Clin Orthop Relat Res 2006;444:120-8.

10. Picada R, Winter RB, Lonstein JE, et al. Postoperative deep wound infection in adults after posterior lumbosacral spine fusion with instrumentation: incidence and management. J Spinal Disord 2000;13:425.

11. Carmouche JJ, Molinari RW. Epidural abscess and discitis complicating instrumented posterior lumbar interbody fusion: a case report. Spine (Phila Pa 1976) 2004;29:E542-6.

12. Ha KY, Kim YH. Postoperative spondylitis after posterior lumbar interbody fusion using cages. Eur Spine J 2004;13:419-24.

13. Maruo K, Berven SH. Outcome and treatment of postoperative spine surgical site infections: predictors of treatment success and failure. J Orthop Sci 2014; 19:398-404.

14. Sierra-Hoffman M, Jinadatha C, Carpenter JL, Rahm M. Postoperative instrumented spine infections: a retrospective review. South Med J 2010;103:25-30.

15. Uckay I, Dinh A, Vauthey L, et al. Spondylodiscitis due to Propionibacterium acnes: report of twentynine cases and a review of the literature. Clin Microbiol Infect 2010;16:353-8.

16. Harris AE, Hennicke C, Byers K, Welch WC. Postoperative discitis due to Propionibacterium acnes: a case report and review of the literature. Surg Neurol 2005;63:538-41.

17. Esteban J, Calvo R, Pardeiro M, Soriano F. Nosocomial diskitis due to Propionibacterium acnes. J Hosp Infect 1998;39:77-8.

18. Albert HB, Lambert P, Rollason J, et al. Does nuclear tissue infected with bacteria following disc herniations lead to Modic changes in the adjacent vertebrae? Eur Spine J 2013;22:690-6.

19. Albert HB, Sorensen JS, Christensen BS, Manniche 
C. Antibiotic treatment in patients with chronic low back pain and vertebral bone edema (Modic type 1 changes): a double-blind randomized clinical controlled trial of efficacy. Eur Spine J 2013;22:697-707.

20. Haidar R, Najjar M, Der Boghossian A, Tabbarah Z. Propionibacterium acnes causing delayed postoperative spine infection: review. Scand J Infect Dis 2010; 42:405-11.

21. Mhaidli HH, Der-Boghossian AH, Haidar RK. Propionibacterium acnes delayed infection following spinal surgery with instrumentation. Musculoskelet Surg 2013;97:85-7.

22. Carricajo A, Nuti C, Aubert E, et al. Propionibacterium acnes contamination in lumbar disc surgery. J Hosp Infect 2007;66:275-7.

23. Stirling A, Worthington T, Rafiq M, Lambert PA, Elliott TS. Association between sciatica and Propionibacterium acnes. Lancet 2001;357:2024-5.

24. Hedequist D, Haugen A, Hresko T, Emans J. Failure of attempted implant retention in spinal deformity delayed surgical site infections. Spine (Phila Pa 1976) 2009;34:60-4.
25. Calvert G, May LA, Theiss S. Use of permanently placed metal expandable cages for vertebral body reconstruction in the surgical treatment of spondylodiscitis. Orthopedics 2014;37:e536-42.

26. Pee YH, Park JD, Choi YG, Lee SH. Anterior debridement and fusion followed by posterior pedicle screw fixation in pyogenic spondylodiscitis: autologous iliac bone strut versus cage. J Neurosurg Spine 2008; 8:405-12.

27. Robinson Y, Tschoeke SK, Kayser R, Boehm H, Heyde CE. Reconstruction of large defects in vertebral osteomyelitis with expandable titanium cages. Int Orthop 2009;33:745-9.

28. Sundararaj GD, Amritanand R, Venkatesh K, Arockiaraj J. The use of titanium mesh cages in the reconstruction of anterior column defects in active spinal infections: can we rest the crest? Asian Spine J 2011;5:155-61.

29. Tokuhashi Y, Ajiro Y, Umezawa N. Conservative follow-up after epidural abscess and diskitis complicating instrumented metal interbody cage. Orthopedics 2008;31:611. 